\section{Adaptação transcultural para o Brasil do instrumento Caregiver Abuse Screen (CASE) para detecção de violência de cuidadores contra idosos}

\author{
Cross-cultural adaptation to Brazil of the \\ instrument Caregiver Abuse Screen (CASE) for \\ detection of abuse of the elderly by caregivers
}

\author{
${ }^{1}$ Hospital Universitário \\ Clementino Fraga Filho, \\ Universidade Federal do \\ Rio de Janeiro, Rio de Janeiro, \\ Brasil. \\ 2 Instituto de Medicina \\ Social, Universidade do \\ Estado do Rio de Janeiro, \\ Rio de Janeiro, Brasil. \\ 3 Faculdade de Medicina, \\ Universidade Estácio de Sá, \\ Rio de Janeiro, Brasil. \\ ${ }^{4}$ Escola Nacional de Saúde \\ Pública Sergio Arouca, \\ Fundação Oswaldo Cruz, \\ Rio de Janeiro, Brasil. \\ 5 Universidade Aberta da \\ Terceira Idade, Universidade \\ do Estado do Rio de Janeiro, \\ Rio de Janeiro, Brasil. \\ Correspondência \\ C. M. Paixão Jr. \\ Setor de Geriatria, Hospital \\ Universitário Clementino \\ Fraga Filho, Universidade \\ Federal do Rio de Janeiro. \\ Av. Brigadeiro Trompowski \\ s/n, Rio de Janeiro, $R J$ \\ 21941-590, Brasil. \\ carlospaixaojr@openlink. \\ com.br
}

\begin{abstract}
This first of two papers focuses on the first part in the cross-cultural adaptation of the Portuguese-language version of Caregiver Abuse Screen (CASE), a brief instrument for detecting domestic violence against the elderly. CASE was originally developed in Canada and used to screen violence against the elderly by interviewing their caregivers. Besides a broad literature review, the evaluation of conceptual and item equivalences involved expert discussion groups. Semantic equivalence included the following steps: two translations and respective backtranslations; an evaluation of referential and general (connotative) equivalence between the original instrument and each version; further discussions with experts in order to define the final version; and pre-testing the latter in 40 caregivers of elderly subjects in an outpatient geriatric clinic. It was possible to establish high-quality conceptual, item, and semantic equivalence for the Portuguese-language version. Although the results shown here were encouraging, they should be reevaluated in light of a forthcoming psychometric analysis (measurement equivalence) to be performed by the research group.
\end{abstract}

Domestic Violence; Aged; Caregivers
Carlos Montes Paixão Jr. ${ }^{1}$ Michael Eduardo Reichenheim 2 Cláudia Leite Moraes 2,3 Evandro Silva Freire Coutinho 2,4 Renato P. Veras 2,5

\section{Introdução}

A existência de atos violentos de negligência e maus-tratos contra os indivíduos idosos é um fenômeno que somente nas duas últimas décadas começou a despertar o interesse na comunidade científica 1,2. No âmbito do estudo da violência doméstica, os maus-tratos contra os idosos foram os últimos a ser contemplados como uma questão de saúde pública. Esse fenômeno vem sendo experimentado sob várias facetas em nossa e em outras sociedades ${ }^{3}$. Expressa-se, também, como maus-tratos sofridos no seio da família, abandono em asilos, perda de direitos próprios ao exercício da cidadania, preconceito e, em última instância, ostracismo e exclusão social 4 .

A solidão, o isolamento e a tendência dos indivíduos idosos a não relatar suas adversidades são apenas alguns fatores relacionados à dificuldade em se obterem estimativas de magnitude deste problema ${ }^{5}$. Assim como no contexto das demais formas de violência doméstica, os serviços de saúde parecem ser um lócus privilegiado para a identificação do problema e implementação de estratégias de ação visando ao seu enfrentamento ${ }^{6}$. Porém, no momento, ainda são poucas as evidências sobre como se deva suspeitar ou reconhecer efetivamente a presença de violência doméstica, e também raros os profissionais de saúde que ativamente rastreiam pacientes que não possuam alguma lesão aparente 7 . Entretanto, há consenso de que instrumentos apropriados de 
rastreamento de violência em idosos sejam cruciais para o progresso da prática e pesquisa desse importante problema de saúde 8 .

Segundo a literatura internacional, a identificação dos maus-tratos contra idosos deve contemplar uma avaliação que utilize fontes diversas de informação como, por exemplo, cuidadores, os próprios idosos e/ou agentes que intervêm nas situações de abuso 9,10. Não é aconselhável que se capte essa informação somente por meio de relatos profissionais ou de informantes eventuais, pois o material gerado por estes tende a supervalorizar a validade de face dos itens que compõem os instrumentos 11. Pessoas idosas, beneficiárias dos cuidados, não estão sempre disponíveis e podem estar acometidas por problemas de saúde, como é o caso de demências que as impedem de fornecer informações. Ademais, podem não desejar relatar o evento do qual foram vítimas, principalmente quando os principais agressores são pessoas de convivência próxima e da família 12 .

Um instrumento de identificação de violência com foco nas informações do cuidador poderia ser útil na prática clínica diária ou em inquéritos epidemiológicos. Alguns estudos indicam que os cuidadores são boas fontes de informação nesse sentido, pois tendem a dar informações confiáveis e complementares, principalmente no caso de idosos com distúrbios cognitivos 11,13. Os cuidadores podem também ser os perpetradores do ato violento. Em um estudo recente de incidência de violência em idosos realizado nos Estados Unidos, verificouse que, em quase $90 \%$ dos casos em que o perpetrador era alguém conhecido, este foi identificado como um membro da família. Em dois terços desses casos, o perpetrador era o esposo(a) ou filho(a) 14 .

Vários autores têm recomendado o trabalho interdisciplinar na avaliação de suspeita ou identificação de violência doméstica contra o idoso $2,15,16,17$. Considerando a indicação cada vez mais presente na prática geriátrica de uma abordagem ampla que vá além da pura investigação de sinais e sintomas clínicos, parece possível e interessante incorporar a primeira avaliação e o atendimento do idoso que é vítima ou está envolvido em maus-tratos nesta abordagem geriátrica global. O treinamento de uma equipe interdisciplinar, já habituada a trabalhar com o idoso, para o rastreio de risco ou a presença de violência pode ser um passo importante na melhoria do processo de detecção de maus-tratos nesta população. Seria, pois, de interesse o estudo de instrumentos que pudessem ser úteis nesse contexto, visando a inseri-los em um ambiente de avaliação geriátrica.
Com base no roteiro proposto por Herdman et al. 18,19 , detalhado na seção seguinte, este artigo enfoca as três primeiras etapas do processo de avaliação da equivalência transcultural entre o instrumento Caregiver Abuse Screen (CASE) concebido por Reis \& Nahmiash 11 em língua inglesa, e uma versão-síntese em português a ser proposta para uso no Brasil.

O CASE foi escolhido a partir de uma revisão de instrumentos de violência em idosos. Nesta, avaliaram-se características psicométricas de diversos instrumentos, freqüência de utilização e adaptabilidade para o contexto cultural brasileiro. Dentre os instrumentos de avaliação do cuidador, o CASE foi o que possuía melhores características, segundo o entendimento de um grupo de expertos consultados 20 . Trata-se de um instrumento breve de autopreenchimento, desenvolvido no Canadá e originalmente concebido para uso na comunidade. Contém perguntas sobre situações que aludem a dimensões de violência física, psicossocial, financeira e negligência, sem, no entanto, argüir diretamente sobre comportamentos ou atos violentos 11 . No estudo original as autoras propuseram que o instrumento fosse aplicado a cuidadores definidos como indivíduos de quem os idosos sistematicamente dependessem para lhes ajudar nas atividades da vida diária.

O histórico psicométrico do CASE indicou uma boa capacidade de discriminação quando avaliado em três grupos de cuidadores, a saber, agressores encaminhados a uma agência de serviços sociais, aqueles sem história de agressão da mesma agência e os da comunidade classificados como não-violentos. A consistência interna revelou um $\alpha=0,71$ na avaliação utilizando os 139 sujeitos dos três grupos 11 . Também há evidência de correlação com outros dois instrumentos já validados para uso em idosos, a saber, o Indicators of Abuse Screen ( $\mathrm{r}=0,41 ; \mathrm{p}<0,001)$ e o HwalekSegstock Elder Abuse Screening Test $(\mathrm{r}=0,26$; $\mathrm{p}<0,025$ ). Ainda que a amostra desse estudo fosse pequena (cerca de cinqüenta cuidadores por grupo) e obtida fora de um ambiente clínico, vale apontar que, pela facilidade de aplicação, as autoras propuseram sua utilização nesse contexto, desde que combinado com outros questionários que também avaliassem as vítimas potenciais ${ }^{11}$.

Os itens do CASE inquirem mais sobre as relações interpessoais e psicológicas do que sobre o contexto social. O instrumento se baseia na teoria de controle 11 , que justifica a violência como uma forma do agressor cercear ações indesejáveis realizadas pela vítima 21 . Na formulação do instrumento, a construção das frases de cada item também é particularmente consistente com a teoria de neutralização, pois justifica e raciona- 
liza os atos violentos pela perspectiva do agressor 22. Esta faz com que o CASE não siga aquela de outros instrumentos de captação de violência que, por vezes, tendem a ser implicitamente críticos em relação ao agressor 13 , encorajando-o a dar respostas socialmente mais aceitáveis. A teoria de neutralização justifica o porquê dos cuidadores serem freqüentemente descritos como surpreendentemente tranqüilos sobre seus comportamentos e por que não se importam em completar os itens do CASE 11.

O instrumento contém apenas oito itens e é de fácil administração. As categorias de resposta estão na forma dicotomizada visando a evitar neutralidade por parte do respondente. Pela sua brevidade, simplicidade e boa aceitabilidade evidenciada no estudo original, o CASE pode ser utilizado como um instrumento de rastreamento de violência doméstica contra o idoso, valendose de entrevistadores sem muita experiência ou treinamento prévio específico. Na prática clínica, pode servir como um primeiro alerta, talvez em combinação com outras ferramentas. Considerando uma pontuação máxima de 8 , análises psicométricas indicaram que escores de 4 ou mais poderiam ser sugestivos de risco aumentado de perpetração de violência, indicando necessidade de investigação mais minuciosa 11 .

O desenvolvimento do CASE é relativamente recente. Até o momento, no melhor do conhecimento dos autores, se dispõe apenas da edição original em inglês, para uso precípuo no Canadá. Diante da insuficiência de ferramentas disponíveis em português para a detecção de violência em idosos, parece oportuno e relevante tornar disponíveis versões lusófonas de instrumentos oriundos de programas de investigação robustos e bem estabelecidos. No contexto brasileiro, o desenvolvimento de pesquisas nessa área se torna ainda mais urgente em função do esforço demandado para construção e avaliação de qualidade de novos instrumentos de aferição, tanto no âmbito clínico como no epidemiológico. Dessa forma, o objetivo do artigo é descrever o processo de adaptação transcultural do CASE, com base em um roteiro formal e estruturado que focaliza especificamente a equivalência conceitual, de itens e semântica entre o instrumento original e uma versão em português 18,19,23.

\section{Métodos}

\section{Um modelo de adaptação transcultural}

O modelo escolhido para a adaptação do CASE à cultura brasileira segue a proposição de Herdman et al. 19. Esses autores utilizam uma aborda- gem que admite a possibilidade de interlocução, mas não assume $a$ priori a equivalência entre construtos nas diferentes culturas. O modelo é calcado na apreciação de diferentes tipos de equivalência. Segundo os autores, somente após percorrer os diferentes aspectos de equivalência apontados por eles, é possível declarar que está estabelecida a adaptação transcultural da nova versão.

\section{Avaliação da equivalência \\ conceitual e de itens}

Após o processo inicial de escolha do CASE, que envolveu revisão bibliográfica sistemática 24 , um grupo de expertos composto por cinco profissionais que atuam nas áreas de violência doméstica, geriatria, epidemiologia e validação de instrumentos avaliou a equivalência conceitual. Como substrato para as discussões, utilizou-se a classificação proposta pelo Ministério da Saúde, comparando-se esta aos conceitos das diversas formas de violência utilizados no Canadá ${ }^{8}$. Outro objetivo do grupo de expertos foi o de explorar se as diferentes dimensões abarcadas pelo instrumento original na definição e apreensão da violência doméstica seriam relevantes em nosso meio. No processo, avaliou-se a pertinência dos itens do instrumento original para a captação de cada uma dessas dimensões da violência doméstica contra o idoso no Brasil. A discussão focalizou o contexto brasileiro e, no tocante aos itens do CASE, se a teoria de neutralização se adequaria à cultura-alvo. Também foi debatido se apenas esse instrumento seria suficiente para a captação de violência sem que houvesse necessidade de outro que investigasse, por exemplo, o idoso, levando-se em conta o seu ambiente de avaliação funcional. Procurou-se observar a capacidade dos itens em abarcar as dimensões descritas no artigo original. Adicionalmente, o grupo questionou se deveriam ser acrescidos itens que identificassem outras dimensões não contempladas.

\section{Avaliação da equivalência semântica}

A avaliação de equivalência semântica envolveu cinco etapas. A inicial consistiu em duas traduções independentes e paralelas do instrumento original em inglês para o português (T1 e T2). Foram utilizados dois profissionais formados em letras, atuantes como tradutores juramentados e, ainda que nascidos no Brasil, perfeitamente fluentes na língua inglesa. Conforme recomendado por Guillemin et al. 23, foram-lhes previamente descritos os objetivos do estudo e a população-alvo. 
Na segunda etapa, T1 e T2 foram retraduzidas por outros dois tradutores juramentados (R1 e R2), fluentes em português, sendo o inglês a língua materna de um deles. As retraduções ocorreram novamente de forma independente e, desta feita, mascaradas em relação ao perfil profissional dos que atuaram na primeira etapa. Como antes, os objetivos do estudo e população-alvo foram apresentados a estes profissionais.

Uma subseqüente avaliação formal foi realizada por um quinto profissional, perfeitamente proficiente nos dois idiomas e também ligado à área de Saúde Coletiva (etapa 3). Nessa avaliação o instrumento adaptado foi comparado ao original sob duas óticas distintas. Deve-se ressaltar que, para cada aspecto de equivalência avaliado, foi usado um formulário específico, desenhado de forma a dissimular a origem dos itens sob escrutínio, i.e., se do original ou da retradução.

Primeiro, avaliou-se a equivalência entre o original e as retraduções sob a perspectiva do significado referencial dos termos/palavras constituintes 19 . O significado referencial concerne às idéias ou objetos do mundo a que uma ou várias palavras aludem. Se há o mesmo significado de uma palavra no original e na respectiva retradução, presume-se que exista uma correspondência literal entre estas. No formulário usado para apreciar o significado referencial, optou-se por escalas analógicas visuais (visual analogue scales) ${ }^{25}$. Assim, a equivalência entre pares de assertivas pôde ser julgada de forma contínua, entre 0 e $100 \%$.

O segundo aspecto apreciado envolveu o significado geral de cada item, contrastando-se o que foi captado na tradução com o original em inglês. Essa correspondência transcende a literalidade das palavras, englobando também aspectos mais sutis, como o impacto que um termo tem no contexto cultural da populaçãoalvo. A apreciação é necessária porque a correspondência literal de um termo não implica que a mesma reação emocional ou afetiva seja evocada em diferentes culturas. É indispensável uma sintonia fina que alcance também uma correspondência de percepção e impacto no respondente. Esta questão é particularmente relevante em relação aos instrumentos usados no âmbito da violência doméstica, pois uma palavra ou assertiva usada com a intenção de agredir psicologicamente pode ter uma grande capacidade de insulto no contexto e local de origem. Por outro lado, a versão literal do termo, usada agora em um novo contexto sócio-cultural, pode carecer de poder afrontador e não ser tão ofensiva. Nesse caso, uma substituição por outro termo permitiria resgatar plenamente a equivalência desejada. Uma primeira etapa de apreciação de significado geral consistiu, portanto, da comparação via formulário abaixo descrito entre R1 e R2 com o instrumento original. No formulário usado para a avaliação de significado geral, utilizou-se uma qualificação em quatro níveis, a saber: inalterado, pouco alterado, muito alterado ou completamente alterado.

Deve-se acrescentar que as situações de divergência entre as avaliações de significados referencial e geral foram explicitamente debatidas, uma vez que desta discussão participou também o avaliador independente.

Além desse profissional, a quarta etapa da avaliação semântica envolveu o mesmo grupo de especialistas que participou da avaliação da equivalência conceitual e itens, tendo como objetivo identificar e encaminhar os problemas de cada uma das etapas pregressas. Nesse momento, a avaliação de significados geral e referencial foi aprofundada, pois o instrumento original foi contrastado tanto com R1 e R2 e as avaliações prévias do quinto tradutor foram debatidas pelo grupo. A partir daí, pôde-se propor uma versão-síntese, ora incorporando itens oriundos de uma das duas versões trabalhadas, ora optandose por certas modificações para melhor atender aos critérios expostos acima.

A quinta e última etapa do processo envolveu um pré-teste da versão-síntese proposta. O objetivo foi testar o grau de aceitabilidade da versão brasileira do CASE em uma população ambulatorial de idosos atendidos em serviços geriátricos. Foram selecionados aleatoriamente quarenta cuidadores de idosos que haviam procurado o Ambulatório de Geriatria do Hospital Universitário, Universidade Federal do Rio de Janeiro. Os itens da versão lhes foram lidos, solicitando que parafraseassem cada pergunta 23 . Optou-se por usar entrevistadores em vez de autopreenchimento, para facilitar a participação dos cuidadores. A cada item do instrumento posto a teste, foi acrescentada uma opção de resposta para que se anotasse se as questões eram culturalmente incompatíveis ou meramente incompreendidas. Os itens que porventura não fossem compreendidos por uma parte do grupo, que, para efeitos práticos, arbitrou-se em $15 \%$ dos idosos entrevistados, voltariam a ser discutidos pelo grupo de expertos. Uma vez implementadas as alterações devidas, a nova versão-síntese integral, se fosse o caso, seria reaplicada a um novo lote de quarenta cuidadores até que todos os itens passassem pelos critérios estipulados. 


\section{Resultados}

Avaliação da equivalência conceitual e de itens

As discussões do grupo de especialistas, municiadas também de consultas à bibliografia temática, sugeriram que os conceitos relacionados à violência doméstica utilizados para a elaboração do instrumento original eram pertinentes ao contexto cultural brasileiro, principalmente no âmbito de populações urbanas. $\mathrm{O}$ instrumento avalia as dimensões de violência física, psicossocial, financeira e negligência, como já exposto na Introdução. Entretanto, o grupo notou que o instrumento deixava de avaliar algumas dimensões importantes como autonegligência, abandono e violência sexual. Ademais, apontaram que o instrumento não se referia diretamente a questões específicas de violência, ainda que isto estivesse em acordo com o proposto pelas autoras no artigo original. Mesmo assim, foi possível identificar validade de face.

A proposta de Reis \& Nahmiash 11 do CASE conter perguntas sutis sobre violência pareceu interessante. Também foi enaltecida a abrangência do instrumento para além de uma única dimensão relacionada à violência doméstica. A fácil utilização e treinamento foram considerados fatores importantes que se coadunavam com o objetivo de uso do CASE em ambientes de atendimento ao paciente idoso não especializados em violência.

\section{Avaliação da equivalência semântica}

Como um todo, a comparação entre os itens oriundos das retraduções (R1 e R2) e os do instrumento original evidenciou uma boa equivalência. Como mostra a Tabela 1, somente dois dos oito itens de R1 e R2 apresentaram um grau de equivalência de significado referencial aquém de $90 \%$ vis à vis ao original. O item mais discrepante revelou-se em R2, especificamente no enunciado do instrumento. O problema teve origem em T2, por conta da tradução dos termos helper e caregiver, vertidos para monitor e assistant, respectivamente. No item 4, por sua vez, o problema aconteceu na passagem de $\mathrm{T} 1$ a $\mathrm{R} 1$, em que a palavra rough foi retraduzida como force. Ainda assim, avaliou-se que o significado geral se manteve inalterado em grande parte das assertivas em T1, principalmente no tocante à primeira retradução. Nesse aspecto da equivalência semântica, constatou-se uma discreta primazia dessa versão sobre a segunda.

Na quarta etapa da avaliação verificaram-se algumas discrepâncias relativas aos significados referencial e geral entre os itens, todas atribuídas a entraves ocorridos na etapa de retradução. Alguns termos traduzidos foram considerados pelo grupo de expertos e o quinto tradutor muito formais para a compreensão fácil pelos indivíduos a serem estudados na cultura-alvo, uma vez que esta possui, em média, níveis baixos ou intermediários de escolaridade.

Ainda que, grosso modo, a etapa anterior tenha endossado ambas as traduções, uma avaliação minuciosa levou a se optar mais pelos itens oriundos de T1 para a elaboração da versão-síntese. Desta, foram incorporados o enunciado e quatro itens (i-1, i-2, i-4, i-5 e i-7). Os demais foram aproveitados de T2 (i-3 e i-6) ou de uma composição entre as duas traduções (i-8).

Na Tabela 2, são apresentadas as traduções dos oito itens e do enunciado do CASE, destacando-se sua origem (T1 ou T2). A Tabela 3 mostra a versão-síntese a partir da qual é possível identificar as modificações realizadas durante a quarta etapa. Estas tiveram diferentes objetivos. A maioria das alterações pretendeu tornar a versão em português mais coloquial e aceitável para a população-alvo. A palavra "freqüentemente", por ser longa e menos usual na linguagem cotidiana, foi sistematicamente substituída por "muitas vezes". Ao pronome "você" foi oferecida a opção alternativa de "o(a) senhor/senhor(a)", para atender a uma possível diferença etária entre o entrevistador e o(a) respondente.

No primeiro item optou-se por manter a tradução da palavra original aggression por "agressividade", em uma tentativa de ampliação do seu significado geral. Embora não correspondam ao mesmo significado referencial, o geral pareceu adequado e menos contundente do que o termo "agressão", que, de forma genérica, seria a proposta das autoras no CASE original 11 . No enunciado do instrumento, embora T1 corresponda à tradução literal, o grupo de expertos entendeu que os termos "ajudante" e "cuidador" poderiam ser pouco compreensíveis no meio cultural brasileiro. Optou-se por utilizar a expressão "pessoa que ajuda ou cuida", pois, diferente do que significa helper no idioma inglês, "ajudante" possui conotação diversa em várias situações, e a palavra "cuidador" é bem menos usual no Brasil do que o é caregiver na cultura original.

Na quinta e última etapa da avaliação semântica (pré-teste) observou-se que o instrumento teve plena aceitação pelo grupo de cuidadores, que tinha oito anos de estudo em média (IC95\%: $7,7-8,4)$. Não houve recusas na passagem do instrumento até o final. Nenhum respondente o considerou cansativo, como seria de se esperar pelo pequeno número de itens. Em nenhuma das quarenta entrevistas houve mais do que $15 \%$ de 
Comparação entre o original em inglês e as retraduções do instrumento Caregiver Abuse Screen (CASE).

\begin{tabular}{|c|c|c|c|c|c|c|}
\hline Original & T1/R1 & Referencial & Geral & T2/R2 & Referencial & Geral \\
\hline $\begin{array}{l}\text { Please answer the following } \\
\text { questions as a helper } \\
\text { or caregiver: }\end{array}$ & $\begin{array}{c}\text { Please answer the following } \\
\text { questions as a helper or caregiver: }\end{array}$ & 100 & IN & $\begin{array}{l}\text { As a monitor or an assistant, please } \\
\text { answer the following questions: }\end{array}$ & 10 & MA \\
\hline $\begin{array}{l}\text { 1) Do you sometimes have } \\
\text { trouble making ( } \_ \text {) control } \\
\text { his/her temper or aggression? }\end{array}$ & $\begin{array}{l}\text { 1) Do you sometimes find it difficult } \\
\text { to make }(X) \text { control his/her } \\
\text { temper or aggressiveness? }\end{array}$ & 90 & IN & $\begin{array}{l}\text { 1) Do you sometimes find it hard } \\
\text { that }(\square) \text { controls } \\
\text { his/her aggressiveness? }\end{array}$ & 90 & PA \\
\hline $\begin{array}{l}\text { 2) Do you often feel you are being } \\
\text { forced to act out of character or } \\
\text { do things you feel bad about? }\end{array}$ & $\begin{array}{l}\text { 2) Do you often feel forced to act } \\
\text { against your own nature or to } \\
\text { do things that displease you? }\end{array}$ & 100 & IN & $\begin{array}{l}\text { 2) Do you often feel forced to act in } \\
\text { disagreement with your character or } \\
\text { to do things that make you feel bad? }\end{array}$ & 90 & PA \\
\hline $\begin{array}{l}\text { 3) Do you find it difficult to } \\
\text { manage ( } \_ \text {'s behaviour? }\end{array}$ & $\begin{array}{l}\text { 3) Do you find it difficult to } \\
\text { control ( } \_ \text {)'s behavior? }\end{array}$ & 90 & PA & $\begin{array}{l}\text { 3) Do you find it hard to control } \\
\text { ( })^{\prime} \text { 's behavior? }\end{array}$ & 90 & PA \\
\hline $\begin{array}{l}\text { 4) Do you sometimes feel that you } \\
\text { are forced to be rough with ( } \square) \text { ? }\end{array}$ & $\begin{array}{l}\text { 4) Do you sometimes feel compelled } \\
\text { to use force with ( } \square \text { ? }\end{array}$ & 80 & PA & $\begin{array}{l}\text { 4) Do you sometimes feel forced } \\
\text { to act harshly towards ( } \square \text { ? }\end{array}$ & 85 & PA \\
\hline $\begin{array}{l}\text { 5) Do you sometimes feel you can't } \\
\text { do what is really necessary or } \\
\text { what should be done for ( } \square \text { ? }\end{array}$ & $\begin{array}{l}\text { 5) Do you sometimes feel you are } \\
\text { unable to do what is really needed } \\
\text { or what needs to be done for }(\square \text { ? }\end{array}$ & 100 & IN & $\begin{array}{c}\text { 5) Do you sometimes feel you cannot } \\
\text { do what is necessary or should be } \\
\text { done for ( }\end{array}$ & 100 & IN \\
\hline $\begin{array}{l}\text { 6) Do you often feel you have to } \\
\text { reject or ignore ( } \square \text { ? }\end{array}$ & $\begin{array}{l}\text { 6) Do you often feel compelled } \\
\left.\text { to reject or ignore ( } \_\right) \text {? }\end{array}$ & 100 & IN & $\begin{array}{l}\text { 6) Do you often feel that you should } \\
\text { reject or ignore ( } \square) \text { ? }\end{array}$ & 100 & IN \\
\hline $\begin{array}{l}\text { 7) Do you often feel so tired and } \\
\text { exhausted that you cannot } \\
\text { meet ( } \square \text { 's needs? }\end{array}$ & $\begin{array}{l}\text { 7) Do you often feel so tired and } \\
\text { exhausted that you cannot look } \\
\text { after/attend to ( } \_ \text {_'s needs? }\end{array}$ & 90 & IN & $\begin{array}{l}\text { 7) Do you often feel so tired or worn } \\
\text { out that you cannot look after } \\
\text { the needs of ( } \square \text { ? ? }\end{array}$ & 90 & IN \\
\hline $\begin{array}{l}\text { 8) Do you often feel you have } \\
\text { to yell at ( }() \text { ? }\end{array}$ & $\begin{array}{l}\text { 8) Do you often feel compelled } \\
\text { to shout at }(\square) \text { ? }\end{array}$ & 100 & IN & $\begin{array}{l}\text { 8) Do you often feel that you } \\
\text { must shout at ( } \square \text { ? }\end{array}$ & 100 & IN \\
\hline
\end{tabular}

Referencial: significado referencial (porcentagem de semelhança); Geral: significado geral; T1/R1: tradução 1/retradução 1; T2/R2: tradução 2/retradução 2; IN: inalterado; PA: pouco alterado; MA: muito alterado; CA: completamente alterado.

itens mal compreendidos, nem houve qualquer sugestão digna de nota por parte dos entrevistados, para os itens, permanecendo inalterada, portanto, a versão já proposta na quarta etapa e mostrada na Tabela 3.

\section{Discussão}

Como já frisado na Introdução, a violência doméstica carece de dados robustos sobre incidência e prevalência no Brasil, mas, com base em estudos realizados em outros países 14,26,27,28, sabe-se que a violência contra os idosos existe e se manifesta sob diferentes formas. Intervenções tendem a ser mais eficientes e eficazes se a detecção é precoce, por meio da coleta de informações oriundas de cuidadores, vizinhos, familiares e outras pessoas que convivem com os idosos 15 .
O CASE atende a essa prerrogativa ao oferecer informações valiosas a partir do cuidador do paciente idoso, avaliando comportamentos relacionados a atos de diversas formas de violência. Não se pode, entretanto, superestimar um único instrumento de coleta de informações de violência doméstica contra o idoso, nem tampouco um único método que, neste caso, se refere a instrumentos de rastreamento, uma vez que há discussões e dúvidas importantes sobre esta questão, até o momento 29.

O modelo seguido neste trabalho foi escolhido por ser abrangente e enfatizar a necessidade de apreciação de diferentes nuanças na adaptação transcultural. Contudo, importa destacar que esse é apenas um dentre vários roteiros disponíveis na literatura afim 19,23,30,31,32. É fundamental, pois, que se ponha à crítica cada uma das etapas envolvidas. A explicitação pormenorizada dos 
Alterações na quarta etapa de equivalência semântica do instrumento Caregiver Abuse Screen (CASE).

\begin{tabular}{|c|c|c|}
\hline \multicolumn{2}{|c|}{ Item escolhido } & Origem \\
\hline \multicolumn{2}{|r|}{ Por favor, responda às seguintes perguntas como ajudante ou cuidador: } & $(\mathrm{T} 1)$ \\
\hline 1) & Você às vezes encontra dificuldades em fazer com que $(\square$ ) controle sua irritação ou agressividade? & 1) (T1) \\
\hline 2) & Você freqüentemente se sente forçado a agir contra sua própria natureza ou a fazer coisas que Ihe desagradam? & 2) (T1) \\
\hline 3) & Você acha difícil controlar o comportamento de ( $\_$? & 3) (T2) \\
\hline 4) & Você às vezes se sente forçado a ser bruto com $(\square)$ ? & 4) (T1) \\
\hline 5) & Você às vezes sente que não consegue fazer o que é realmente necessário ou o que deve ser feito para $(\square)$ ? & 5) (T1) \\
\hline 6) & Você freqüentemente sente que deve rejeitar ou ignorar $(\square)$ ? & 6) (T2) \\
\hline 7) & Você freqüentemente se sente tão cansado e exausto que não consegue atender às necessidades de $(\square)$ ? & 7) (T1) \\
\hline 8) & Você freqüentemente sente que deve gritar com () ? & 8) $(\mathrm{T} 1+\mathrm{T} 2)$ \\
\hline
\end{tabular}

Versão em português do instrumento Caregiver Abuse Screen (CASE) utilizado na suspeição de violência de cuidadores contra indivíduos idosos.

\begin{tabular}{|c|c|c|}
\hline Sendo a pessoa que ajuda ou cuida, por favor, responda sim ou não às seguintes perguntas & Sim & Não \\
\hline 1) V/S às vezes encontra dificuldade em fazer com que $\left(\_\right.$) controle sua irritação ou agressividade? & - & 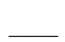 \\
\hline 2) V/S muitas vezes se sente forçado(a) a agir contra sua própria natureza ou a fazer coisas que lhe desagradam? & & 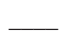 \\
\hline 3) V/S acha difícil controlar o comportamento de $(\square)$ ? & - & $\ldots$ \\
\hline 4) V/S às vezes se sente forçado(a) a ser bruto(a) com ( $\square$ ) & - & - \\
\hline 5) V/S às vezes sente que não consegue fazer o que é realmente necessário ou o que deve ser feito para ( $)$ ? & - & - \\
\hline 6) V/S muitas vezes acha que tem de rejeitar ou ignorar $(\square)$ ? & $\underline{-}$ & - \\
\hline 7) V/S muitas vezes se sente tão cansado(a) e exausto(a) que não consegue dar conta das necessidades de ( $\square$ )? & - & - \\
\hline 8) V/S muitas vezes acha que tem de gritar $\operatorname{com}(\square)$ ? & & 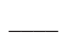 \\
\hline
\end{tabular}

passos realizados, dos dilemas surgidos ao longo do processo e a experiência acumulada durante a adaptação do CASE pretendem enriquecer esse debate.

Apesar da constatação de equivalência conceitual, nas várias etapas de atuação do grupo de especialistas aventou-se a possibilidade de inclusão de outras dimensões da violência em idosos na versão a ser usada no Brasil. Como visto, o CASE deixa de avaliar algumas dimensões importantes, como autonegligência, abandono e violência sexual. Entretanto, um problema conceitual importante reside na inabilidade atual dos que estudam os diversos matizes de violência doméstica em idosos em definir os atributos do que seja um relacionamento normal, apropriado e adequado 33. Os estudiosos, tampouco, conseguiram estabelecer padrões de cuidado sobre os quais os clínicos pudessem se basear para determinar com certa segurança se o idoso estaria ou não recebendo cuidados minimamente adequa- dos. Por exemplo, a falha em ministrar medicação ao idoso pode bem ser definida como negligência, mas vários profissionais não seriam tão rígidos em relação a esta atitude, se eventual.

Também, o conceito de autonegligência dá margem a discussões sobre sua identificação como violência na acepção da palavra 34 e, logo, é de difícil avaliação. Alguns idosos com atitudes de autonegligência podem não reconhecer que suas ações são perniciosas e talvez prefiram o estilo de vida que adotaram desde mais jovens. Além disso, uma avaliação de autonegligência deve passar preferencialmente por uma avaliação domiciliar, algo que está fora do contexto de avaliação geriátrica ampla ambulatorial. A noção de abandono, que envolve isolamento, também torna limitada sua avaliação em locais fora do domicílio. Apenas um instrumento encontrado na revisão bibliográfica avalia violência sexual (Carer Abuse Assessment Protocol for Nurses) 35. Este, porém, propõe uma abordagem eminen- 
temente qualitativa e que, por requerer um treinamento prévio em violência doméstica por parte do avaliador, se prestaria pouco ao que se pretende no contexto da avaliação funcional do idoso. Nenhum instrumento encontrado avalia abandono ou autonegligência. De toda a sorte, essas questões merecem ser contempladas em discussões futuras sobre instrumentos de avaliação do cuidador. Uma alternativa ao uso de um instrumento que abarcasse todas as dimensões de violência doméstica seria a junção de dois instrumentos de avaliação, um contemplando o aspecto do cuidador e outro o do idoso.

Em relação à primeira etapa de equivalência semântica, o perfil dos tradutores também importa, como apontam vários autores ocupados com processos de adaptação de instrumentos 19,23,31. Segundo estes, é importante que o processo de tradução seja realizado por profissionais cuja língua-mãe e cultura sejam aquelas para as quais está se realizando a tradução. Isso significa que, idealmente, para T1 e T2, os tradutores devessem ser brasileiros com domínio da língua inglesa e, no caso das duas retraduções do CASE, que fossem canadenses anglófonos com bom domínio do português. Um dos profissionais utilizados nas retraduções era estadunidense (R1), que, por ter um perfil bastante próximo ao recomendado, deve ter contribuído positivamente no produto final. Segundo Guillemin et al. 23, deve haver tantas retraduções quantas traduções e, de preferência, que os dois processos sejam realizados por equipes de tradutores e não apenas um para cada tradução e retradução. Entretanto, segundo os mesmos autores, questões situacionais e financeiras podem não permitir essa organização. Este foi o caso no processo do CASE, em que dois tradutores e o mesmo número de retradutores foram utilizados. Não houve equipes de tradutores, mas a discussão da quarta etapa serviu para identificar, como já antes mencionado, problemas nas traduções e adequação à população-alvo.

Seguindo uma outra recomendação de Guillemin et al. 23 e Herdman et al. 19, interessa que os tradutores tenham conhecimento do que trata o instrumento, da população-alvo para a qual este será adaptado e quais as suas dimensões. Por outro lado, aos retradutores devem ser dadas apenas explicações limitadas sobre o contexto, evitando que estes utilizem seu conhecimento prévio para corrigir erros eventuais no processo inicial de tradução. Também estes cuidados foram tomados no processo de adaptação do CASE. Possivelmente, também este cuidado foi importante para que a versão-síntese do instrumento requeresse poucas alterações na fase de discussão.
Houve, no enunciado, um problema de identificação da palavra helper/caregiver em T2. O profissional responsável por T1 havia utilizado, no entanto, os termos corretos em português. A existência de mais de um par de tradução-retradução (T1/R1 - T2/R2) facilitou a tomada de decisões para a elaboração da versão-síntese. Porém, uma recomendação genérica sobre o número ideal de pares não parece interessante, na medida em que esta decisão está intimamente ligada ao custo-benefício do processo de cada pesquisa. A questão sobre o número ótimo de tradutores poderia ser oportunamente respondida com o incremento de programas de investigação que avaliem a efetividade das diferentes estratégias de adaptação transcultural.

Foi grande a influência da quarta etapa na elaboração da versão-síntese, tanto no ajuste final para as escolhas entre expressões semelhantes oriundas das duas traduções, quanto para lidar com problemas processuais. Por exemplo, o problema ventilado anteriormente, causado pelo fato de um dos profissionais envolvidos na etapa de retradução não ter o inglês como língua-mater pôde ser contornado por meio dessa avaliação mais detalhada. A importância de uma avaliação final interdisciplinar nos processos de adaptação não deve ser menosprezada.

A decisão de adaptar a versão-síntese para que o CASE, originalmente um instrumento de autopreenchimento, fosse utilizado através de perguntas realizadas por entrevistador se baseou na constatação que, em função da baixa escolaridade de grande parte da população brasileira, as pesquisas na área de saúde costumam dar prioridade a entrevistas face a face na coleta de informações. No entanto, não se deve perder a perspectiva de que, genericamente, processos de aferição baseados em autopreenchimento podem ser mais acurados, uma vez que não há o constrangimento de se expor oralmente questões sensíveis e há sempre a possibilidade de se garantir anonimato 29,36. Essas questões são ainda mais relevantes no contexto da violência doméstica, naturalmente propensa a ser velada. Ainda assim, a forma como são formuladas as perguntas do CASE levou o grupo de expertos a concluir que o instrumento se prestaria tanto a uma quanto a outra forma de aferição, indicando, deste modo, uma aceitável equivalência operacional.

É possível que a composição multidisciplinar do grupo de expertos, também bilíngüe, e a utilização de um quinto tradutor com experiência na área de saúde coletiva tenha auxiliado bastante no processo de adaptação dos itens traduzidos. Estes cuidados tornaram a linguagem do CASE mais inteligível e amistosa à po- 
pulação-alvo, facilitando sua plena aceitação e compreensão.

A partir da avaliação da equivalência conceitual, de itens e semântica foi possível propor uma primeira versão em português do CASE. No entanto, ainda restam alguns passos para se aceitar com certa segurança que a equivalência transcultural entre o original e a versão tenha sido plenamente alcançada. Conforme exposto na Introdução, a equivalência de mensuração, estudada por meio da comparação entre as propriedades psicométricas da versão em português e do instrumento original, é mais uma tarefa importante nesse processo e deverá ser realizada no futuro próximo. Além disso, é fundamental que esta primeira versão seja posta à crítica pelos profissionais interessados. A vasta gama de detalhes e opções, muitas delas intrinsecamente subjetivas, demanda que o aprimoramento do instrumento adaptado dependa de debates e negociações contínuas entre pares. Vale lembrar que somente após a etapa de equivalência de mensuração se poderá atestar a equivalência operacional e, conseqüentemente, propor uma equivalência funcional 18,19 . Somente aí será seguro utilizar o CASE rotineiramente como ferramenta de suspeita de violência doméstica envolvendo o indivíduo idoso e seu cuidador, par e passo com outros instrumentos de rastreamento que avaliem diretamente o indivíduo idoso.

\section{Resumo}

Este artigo descreve a primeira parte da adaptação transcultural da versão em português, para o Brasil, do Caregiver Abuse Screen (CASE), um instrumento simplificado para suspeição de violência contra o idoso. O CASE foi originalmente desenvolvido no Canadá e utilizado para rastrear violências em idosos entrevistando seus cuidadores. O processo de avaliação de equivalências conceitual e de itens, que envolveu uma ampla e sistemática revisão bibliográfica, consistiu de uma discussão em grupo de expertos. A equivalência semântica envolveu duas traduções e respectivas retraduções em paralelo; uma avaliação de equivalência de significados referencial e geral entre o CASE original e as versões em português; discussões posteriores com o grupo de expertos para definir a versão final; $e$ um pré-teste com quarenta cuidadores de pacientes idosos em um serviço de atendimento ambulatorial de geriatria. Foi possível estabelecer uma versão em português para o Brasil com boa qualidade de equivalência conceitual, de itens e semântica. Embora os resultados aqui descritos sejam encorajadores, eles devem ser reavaliados à luz de evidências psicométricas (equivalência de mensuração) que oportunamente serão apresentadas por este grupo de estudo.

\section{Colaboradores}

C. M. Paixão Jr. participou da escolha do tema, organização do trabalho, revisão dos instrumentos, organização do grupo de expertos e do próprio grupo de expertos, no pré-teste, montagem das tabelas, procura e revisão das publicações, discussão, organização e edição final do artigo. M. E. Reichenheim contribuiu na escolha do tema, organização do trabalho, com a participação no grupo de expertos, discussão, organização e edição final do artigo. C. L. Moraes, E. S. F. Coutinho e R. P. Veras participaram do grupo de expertos e colaboraram na discussão, organização e edição final do artigo.

\section{Agradecimentos}

C. M. Paixão Jr. foi apoiado pela Fundação de Amparo à Pesquisa do Estado do Rio de Janeiro (FAPERJ), processo no. E-26/150.727/2002. M. E. Reichenheim foi parcialmente apoiado pelo Conselho Nacional de Desenvolvimento Científico e Tecnológico (CNPq), processo no. 306939-2003-7. Os autores agradecem a valiosa contribuição de A. C. C. Pinto, C. Bereicôa, L. M. Martins, B. C. Lima nas entrevistas de campo. 


\section{Referências}

1. Krug EG, Dahlberg LL, Mercy JA, Zwi AB, Lozano R. World report on violence and health. Geneva: World Health Organization; 2002.

2. Machado L, Queiroz ZV. Negligência e maus-tratos. In: Freitas EV, Py L, Neri AL, Cançado FAXC, Gorzoni ML, Doll J, organizadores. Tratado de geriatria e gerontologia. Rio de Janeiro: Editora Guanabara Koogan; 2002. p. 791-7.

3. Barnett OW, Miller-Perrin CL, Perrin RD. History and definitions. In: Barnett O, Miller-Perrin C, Perrin $\mathrm{RD}$, editors. Family violence across the lifespan: an introduction. London: Sage Publications; 1997. p. 3-17.

4. Queiroz ZPV. Violência contra a velhice: considerações preliminares sobre uma nova questão social. Mundo Saúde 1997; 21:204-7.

5. Kosberg JI. Preventing elder abuse: identification of high risk factors prior to placement decisions. Gerontologist 1988; 28:43-50.

6. American Medical Association Council on Scientific Affairs. Diagnostic and treatment guidelines concerning child abuse and neglect. Chicago: American Medical Association; 1984.

7. Rathbone-McCuan E, Voyles B. Case detection of abused elderly parents. Am J Psychiatry 1982; 139:189-92.

8. Brasil. Portaria n ${ }^{\circ} .737$ de 16 de maio de 2001. Política nacional de redução da morbimortalidade por acidentes e violências. Diário Oficial da União 2001; 18 mai.

9. Spencer C. New direction for research on interventions with abused older adults. In: MacLean MJ, editor. Abuse and neglect of all the Canadians: strategies for change. Toronto: Thompson Educational Inc.; 1995. p. 143-58.

10. Carp FM. Some studies needed to test and improve the model. In: Carp FM, editor. Elder abuse in the family: an interdisciplinary model for research. New York: Springer Publishing Company; 2000. p. 165-81.

11. Reis M, Nahmiash D. Validation of the Caregiver Abuse Screen (CASE). Can J Aging 1995; 14:45-60.

12. Gordon RM, Brill D. The abuse and neglect of the elderly. Int J Law Psychiatry 2001; 24:183-97.

13. Sengstock MC, Hwalek M. A review and analysis of measures for the identification of elder abuse. J Gerontol Soc Work 1987; 10:21-36.

14. National Center on Elder Abuse. The National Elder Abuse Incidence Study: final report. Washington DC: National Center on Elder Abuse; 1998.

15. Decalmer P. Clinical presentation and management. In: Decalmer P, Glendenning F, editors. The mistreatment of elderly people. London: Sage Publications; 1997. p. 42-73.

16. Lachs MS, Fulmer T. Recognizing elder abuse and neglect. Clin Geriatr Med 1993; 9:665-81.

17. Parkins SM. Hospital response to elder abuse: the adult protective team. In: Baumhover LA, Beall SC, editors. Abuse, neglect, and exploitation of older persons: strategies for assessment and intervention. Baltimore: Health Professionals Press; 1996. p. $163-84$
18. Herdman M, Fox-Rushby J, Badia X. "Equivalence" and the translation and adaptation of health-related quality of life questionnaires. Qual Life Res 1997; 6:237-47.

19. Herdman M, Fox-Rushby J, Badia X. A model of equivalence in the cultural adaptation of HRQoL instruments: the universalist approach. Qual Life Res 1998; 7:323-35.

20. Paixão Jr. CM. Violência doméstica: instrumentos de aferição de violência contra o idoso no ambiente da avaliação geriátrica ampla [Tese de Doutorado]. Rio de Janeiro: Instituto de Medicina Social, Universidade do Estado do Rio de Janeiro; 2006.

21. Bendik MF. Reaching the breaking point: dangers of mistreatment in elder caregiving situations. J Elder Abuse Neglect 1992; 4:39-59.

22. Tomita SK. The denial of elder mistreatment by victims and abusers: the application of neutralization theory. Violence Vict 1990; 5:171-84.

23. Guillemin F, Bombardier C, Beaton D. Cross-cultural adaptation of Health-Related Quality of Life measures: literature review and proposed guidelines. J Clin Epidemiol 1993; 46:1417-32.

24. Paixão Jr. CM, Reichenheim ME. Uma revisão sobre instrumentos de rastreamento de violência doméstica contra o idoso. Cad Saúde Pública 2006; 22:1137-49.

25. Streiner DL, Norman GR. Health measurement scales: a practical guide to their development and use. New York: Oxford Medical Publications; 1995.

26. Pillemer KA, Finkelhor D. The prevalence of elder abuse: a random sample survey. Gerontologist 1988; 28:51-7.

27. Pillemer KA, Moore DW. Abuse of patients in nursing homes: findings from a survey of staff. Gerontologist 1989; 29:314-20.

28. Podineks E. National survey on abuse of the elderly in Canada. J Elder Abuse Negl 1992; 4:5-58.

29. Fulmer T, Guadagno L, Dyer CB, Connoly MT. Progress in elder abuse screening and assessment instruments. J Am Geriatr Soc 2004; 52:297-304.

30. Berkanovic E. The effect of inadequate language translation on hispanics' responses to health surveys. Am J Public Health 1980; 70:1273-81.

31. Perneger TV, Leplège A, Etter J-F. Cross-cultural adaptation of a psychometric instrument: two methods compared. J Clin Epidemiol 1999; 52:1037-46.

32. Behling O, Law K. Translating questionnaires and other research instrument: problems and solutions. Thousand Oaks: Sage Publications; 2000.

33. Fulmer T. Elder mistreatment. Annu Rev Nurs Res 2002; 20:369-95.

34. Decalmer P, Glendenning F. The mistreatment of elderly people. London: Sage Publications; 1997.

35. Davies M. Recognizing abuse: an assessment tool for nurses. In: Decalmer P, Glendenning F, editors. The mistreatment of elderly people. London: Sage Publications; 1997. p. 102-6.

36. Pelton LG. Interpreting family violence data. Am J Orthopsychiatry 1979; 49:194, 372-4.

Recebido em 19/Jan/2006

Versão final reapresentada em 15/Fev/2007

Aprovado em 19/Abr/2007 